\title{
Distribution of echocardiographic parameters and their associations with cardiovascular risk factors in the Rotterdam Study
}

\author{
Isabella Kardys · Jaap W. Deckers • \\ Bruno H. Ch. Stricker · Wim B. Vletter • \\ Albert Hofman · Jacqueline Witteman
}

Received: 6 August 2009/Accepted: 23 March 2010/Published online: 22 May 2010

(C) The Author(s) 2010. This article is published with open access at Springerlink.com

\begin{abstract}
Insight into echocardiographic parameters in the general population may facilitate early recognition of ventricular dysfunction, reducing the population morbidity and mortality of heart failure. We examined the distribution of structural, systolic and diastolic echocardiographic parameters and their associations with cardiovascular risk factors in the Rotterdam Study, a population-based cohort study in men and women aged $\geq 55$ years. Participants with prevalent heart failure, myocardial infarction and atrial fibrillation and flutter were excluded. Echocardiographic parameters were assessed using two-dimensional, M-mode and Doppler echocardiography. Echocardiograms were available in 4,425 participants. Structural parameters were generally larger in men, and most consistently associated with age, body mass index and blood pressure in both sexes. Prevalence of moderate or poor left ventricular systolic function was $3.9 \%$ in men and $2.1 \%$ in women. Age, body mass index and blood pressure were most consistently associated with systolic function. E/A ratio was lower in women than in men. Age and diastolic blood pressure were most consistently associated with E/A ratio in both sexes. In conclusion, ventricular systolic and diastolic dysfunction is
\end{abstract}

Electronic supplementary material The online version of this article (doi:10.1007/s10654-010-9453-5) contains supplementary material, which is available to authorized users.

I. Kardys · B. H. Ch. Stricker · A. Hofman · J. Witteman ( $₫)$ Department of Epidemiology, Erasmus Medical Center, P.O. Box 2040, 3000 CA Rotterdam, The Netherlands e-mail: j.witteman@erasmusmc.nl

I. Kardys · J. W. Deckers - W. B. Vletter

Department of Cardiology, Thoraxcenter, Erasmus MC,

Rotterdam, The Netherlands present in asymptomatic individuals. Selected established cardiovascular risk factors are associated with structural, systolic and diastolic parameters.

Keywords Cardiovascular disease - Diastolic function . Echocardiography $\cdot$ Systolic function
Abbreviations
ACE Angiotensin converting enzyme
BMI Body mass index
ECG Electrocardiogram
GP General practitioner
HDL High-density lipoprotein
IVST Interventricular septum thickness
LVED Left ventricular end diastolic dimension
LVES Left ventricular end systolic dimension
PWT Posterior wall thickness

\section{Introduction}

Heart failure is an important health problem. Its prevalence is increasing, in part because of improved treatment of patients with heart failure and increased survival of patients with myocardial infarction [1]. Since heart failure is primarily a condition in the elderly, the aging of the population also contributes to its increasing prevalence. Although in most patients, abnormalities of systolic and diastolic dysfunction coexist [1], it has been shown that persons with normal ejection fraction can have impaired diastolic function [2]. Also of note is, that persons with a very low ejection fraction may be asymptomatic; this has been termed preclinical systolic dysfunction [3]. 
It has been recognized that therapeutic interventions introduced even before the appearance of left ventricular dysfunction or symptoms can reduce the population morbidity and mortality of heart failure $[1,4]$. This underlines the importance of early recognition of preclinical ventricular dysfunction. Echocardiography, which has become a common method to assess ventricular function, may be useful to gain more insight into this matter. To its advantage, it is noninvasive and comprehensive. As a consequence, echocardiography has been used in several population-based studies to examine the prevalence and determinants of systolic and diastolic dysfunction in the community [3, 5-9] and to investigate the predictive value of echocardiographic parameters for heart failure, cardiovascular disease and mortality [3, 7, 10-13].

The growing importance of insight into echocardiographic characteristics of the general population has prompted us to perform echocardiography in the Rotterdam Study, a population-based study in men and women aged 55 years and over. The purpose of the present report is to describe the distribution of structural, systolic and diastolic echocardiographic parameters in our study population and to examine the associations of established cardiovascular risk factors with these echocardiographic parameters.

\section{Methods}

\section{Study population}

The study was performed within the framework of the Rotterdam Study, a population-based cohort study aimed at assessing the occurrence of and risk factors for chronic diseases in the elderly [14]. Baseline visits of the Rotterdam Study took place in 1990-1993. All inhabitants of a suburb of Rotterdam aged 55 years and over were invited and 7,983 agreed to participate (response 78\%). Follow-up visits took place in 1993-1994 and 1997-1999. In 20002001, the cohort was extended with 3,011 participants from the same suburb (response 67\%), also aged 55 years and over. For the present study, data collection took place from 2002-2005. Within this period, the participants from the original cohort completed their fourth center visit ( $n=3,550$ ), and the participants of the extended cohort completed their second center visit $(n=2,389)$. Of these, 3,052 and 2,235, respectively, underwent cardiac echocardiography. The large majority of missing echocardiograms was explained by incidental absence of echocardiographers. Participants with prevalent heart failure $(n=304)$, prevalent myocardial infarction, $(n=561)$, and atrial fibrillation $(n=124)$ or atrial flutter $(n=13)$ at the time of echocardiography were excluded from the analysis. This led to the exclusion of a total of 862 persons and consequently resulted in a total of 4,425 participants available for analysis. The medical ethics committee of Erasmus Medical Center, Rotterdam, approved the study. Participants gave written informed consent and permission to retrieve information from treating physicians. The investigation conforms with the principles outlined in the declaration of Helsinki.

Assessment of cardiovascular risk factors and prevalent disease

A trained interviewer visited all participants at home and collected information on current health status, medical history, drug use, and smoking, using a computerized questionnaire. Participants were categorized as current, past and never smokers. Clinical measures were obtained during a visit at the study center. Height and weight were measured and the body mass index (BMI) was calculated (weight (kg)/ height $\left.(\mathrm{m})^{2}\right)$. Blood pressure was measured at the right brachial artery using a random-zero sphygmomanometer with the participant in sitting position. Serum total cholesterol and high-density lipoprotein (HDL) cholesterol were obtained using an automatic enzymatic procedure (Hitachi 911, Roche CHOD PAP). Diabetes mellitus was defined as the use of antidiabetic medication or a fasting glucose level of $\geq 7 \mathrm{mmol} / \mathrm{l}$ [15]. Presence of symptomatic heart failure at the time of echocardiography was assessed by using a validated score similar to the definition of heart failure of the European Society of Cardiology, as described previously [16], and the information needed was obtained by screening of all medical records of general practitioners (GPs) in retrospect for the occurrence of heart failure and obtaining letters and discharge reports from medical specialists when a case was found. A history of myocardial infarction was considered present when myocardial infarction was detected by screening GP medical records and reviewing letters and discharge reports from medical specialists, or when an ECG characteristic of prior myocardial infarction was detected during a follow-up visit. Presence of atrial fibrillation or atrial flutter at the time of echocardiography was assessed with electrocardiography by applying the Modular ECG Analysis System (MEANS), which has been extensively evaluated $[17,18]$, and is characterized by a high sensitivity (96.6\%) and a high specificity $(99.5 \%)$ in coding arrhythmias [19]. For a small sample of participants, ECGs were not available at the time this report was written because of logistic problems and were approximated by using ECGs from the previous examination (year 2000-2001).

\section{Echocardiography}

For each participant, an echocardiogram was obtained. The first 2,188 echocardiograms were performed with a 
commercially available ultrasonography system (AU3 Partner, Esaote Biomedica, with a $3.5 / 2.5 \mathrm{MHz}$ transducer). The following 3,099 echocardiograms were performed with another commercially available system (Acuson Cypress, with a 3V2c transducer). A standardized protocol was used, including two-dimensional scanning in the parasternal long axis view, parasternal short axis view, apical view and subcostal view, M-mode scanning in the parasternal long axis view, and pulsed wave Doppler scanning in the apical four chamber view [20]. Echocardiograms were recorded onto VHS tape and assessed at the reading center, which was located at Erasmus Medical Center.

Several structural parameters were assessed [21]. Left atrium diameter, left ventricular end systolic dimension (LVES), left ventricular end diastolic dimension (LVED), end diastolic interventricular septum thickness (IVST) and end-diastolic left ventricular posterior wall thickness (PWT) were measured in the parasternal long axis view using M-mode with two-dimensional guidance. Left ventricular mass (grams) was calculated as $0.80(1.04 *$ $\left.(\text { IVST }+ \text { LVED }+ \text { PWT })^{3}-(\text { LVED })^{3}\right)+0.6$, according to Devereux et al [22]. Fractional shortening at the endocardium was calculated as (LVED-LVES)/LVED*100\% [21]. Global left ventricular systolic function was qualitatively assessed, without quantitative measurement, from the twodimensional echocardiogram and classified as normal, fair, moderate or poor. Furthermore, diastolic parameters were measured [23]. Pulsed Doppler recordings of transmitral filling velocity were performed in the apical 4-chamber view, with the sample volume placed in the mitral valve orifice near the tips of the leaflets. Doppler peak E and peak A velocities were averaged over three cycles. E/A ratio was computed by dividing Doppler peak E velocity by Doppler peak A velocity. Early mitral valve velocity deceleration time was measured as the time between the peak E wave and the upper deceleration slope extrapolated to the zero baseline.

Echocardiograms were made and read by four trained echocardiographers. To assess intra-reader and inter-reader agreement, 32 participants were examined in duplicate. For continuous variables, overall median percent intra-reader and inter-reader measurement variabilities were calculated as the absolute measurement difference divided by the average of the two measurements, multiplied by 100 . Overall median intra-reader and inter-reader variabilities for left ventricular end-diastolic dimension were 3 and $4 \%$, respectively. For left ventricular end-systolic dimension both variabilities were $6 \%$, and for fractional shortening both were $8 \%$. Overall median intra-reader and inter-reader variabilities for E/A ratio were 15 and $18 \%$, respectively, and for deceleration time both were $16 \%$. Finally, the percentage agreement for categorization of left ventricular systolic function as normal or other than normal was $82 \%$ within readers and $86 \%$ between readers.

\section{Statistical analysis}

We calculated means, medians and proportions of cardiovascular risk factors and echocardiographic characteristics in men and women. Differences between the sexes were tested with $t$-tests, chi square tests and Mann-Whitney tests where appropriate.

Subsequently, we investigated the association between cardiovascular risk factors and echocardiographic parameters. We performed linear regressions with age, BMI, systolic blood pressure, diastolic blood pressure, total cholesterol, HDL-cholesterol, diabetes mellitus and smoking as independent variables and structural echocardiographic parameters (diameter of left atrium, left ventricular endsystolic dimension, left ventricular end-diastolic dimension, interventricular septum thickness, left ventricular posterior wall thickness, left ventricular mass), systolic parameters (ejection fraction, fractional shortening) and diastolic parameters (mitral valve inflow peak $\mathrm{E}$ velocity, mitral valve inflow peak A velocity, E/A ratio and mitral valve inflow deceleration time) as dependent variables. Continuous independent variables were examined per standard deviation increase. For some of the dependent variables, the distribution of the residuals was skewed. After log-transformation of these variables, the residuals were normally distributed with a constant variance. To make the regression coefficients more informative, units of dependent variables were multiplied by $10^{-2}$. The analysis was performed separately in men and women. We adjusted for age and type of ultrasonography system used, and subsequently, we also adjusted the analysis of each risk factor for all other risk factors and also for use of diuretics, beta-blockers, ACE inhibitors, lipid lowering drugs and heart rate.

We used analysis of covariance to compare risk factors, structural echocardiographic parameters, and fractional shortening according to categories of qualitatively assessed left ventricular systolic function and categories of E/A ratio and diastolic function. Few men and women had poor left ventricular systolic function ( $n=19$ and $n=10$, respectively), therefore we pooled moderate and poor systolic function for the analysis to make the results easier to interpret. We categorized E/A ratio according to cut points used in previous reports [3, 24].

All analyses were performed using SPSS 11.0. Values for cardiovascular covariates were missing in less than $3 \%$ of participants, except for BMI, which was missing in 5.5\%. Missing values were handled by single imputation using the expectation-maximization algorithm in SPSS 11.0. All tests were two-sided. We ran several analyses in duplicate to compare the two ultrasonography systems we used. 


\section{Results}

Table 1 shows the distribution of cardiovascular risk factors in men and women. Women were significantly older than men, although this was a clinically modest difference of 1.1 years. In Table 2, the distribution of echocardiographic characteristics is displayed in men and women. All characteristics showed significant differences across the sexes; in general, structural parameters were larger in men, systolic function was worse in men, and with regard to diastolic function, E/A ratio was lower in women.

For reasons of conciseness, only the multivariable results are presented. Overall, age, BMI, systolic blood pressure and diastolic blood pressure were significantly associated with most structural parameters in men and women (Supplementary Table 1). Age, BMI and systolic
Table 1 Population characteristics
Categorical variables are expressed as percentage. Continuous variables are expressed as mean \pm standard deviation. $T$-tests and chi square tests were used where appropriate

\begin{tabular}{lllc}
\hline Variable & Men $(n=1,736)$ & Women $(n=2,689)$ & $P$-value \\
\hline Age (years) & $70.8 \pm 7.0$ & $71.9 \pm 7.5$ & $<0.001$ \\
Body mass index $\left(\mathrm{kg} / \mathrm{m}^{2}\right)$ & $27.2 \pm 3.4$ & $27.7 \pm 4.5$ & $<0.001$ \\
Systolic blood pressure $(\mathrm{mmHg})$ & $148 \pm 20$ & $151 \pm 22$ & $<0.001$ \\
Diastolic blood pressure (mmHg) & $81 \pm 11$ & $79 \pm 11$ & $<0.001$ \\
Total cholesterol (mmol/l) & $5.4 \pm 0.9$ & $5.9 \pm 0.9$ & $<0.001$ \\
HDL-cholesterol (mmol/l) & $1.3 \pm 0.3$ & $1.6 \pm 0.4$ & $<0.001$ \\
Diabetes mellitus (\%) & 13.4 & 12.7 & 0.49 \\
Smoking & & & $<0.001$ \\
Never (\%) & 13.4 & 43.1 & \\
Former (\%) & 68.2 & 42.8 & $<0.001$ \\
Current (\%) & 18.4 & 14.0 & $<0.001$ \\
Use of diuretics $(\%)$ & 5.4 & 11.8 & 0.79 \\
Use of beta blockers (\%) & 11.9 & 15.6 & 0.52 \\
Use of ACE-inhibitors (\%) & 11.2 & 11.0 & 11.9 \\
Use of lipid-lowering drugs (\%) & 11.2 & & \\
\hline
\end{tabular}

Table 2 Echocardiographic characteristics

\begin{tabular}{|c|c|c|c|}
\hline Variable & Men $(n=1,736)$ & Women $(n=2,689)$ & $P$-value \\
\hline \multicolumn{4}{|l|}{ Structural parameters } \\
\hline Left atrium diameter $(\mathrm{mm})$ & $42 \pm 5$ & $39 \pm 5$ & $<0.001$ \\
\hline Left ventricular end systolic dimension (mm) & $32(29-36)^{\mathrm{a}}$ & $29(27-32)^{\mathrm{a}}$ & $<0.001$ \\
\hline Left ventricular end diastolic dimension (mm) & $53 \pm 5$ & $49 \pm 5$ & $<0.001$ \\
\hline Interventricular septum thickness (mm) & $8(8-10)^{\mathrm{a}}$ & $8(7-9)^{\mathrm{a}}$ & $<0.001$ \\
\hline Left ventricular posterior wall thickness (mm) & $8(7-8)^{\mathrm{a}}$ & $7(7-8)^{\mathrm{a}}$ & $<0.001$ \\
\hline Left ventricular mass $(\mathrm{g})$ & $157(135-187)^{\mathrm{a}}$ & $130(110-151)^{\mathrm{a}}$ & $<0.001$ \\
\hline \multicolumn{4}{|l|}{ Systolic parameters } \\
\hline Ejection fraction $(\%)$ & $0.77(0.71-0.82)^{\mathrm{a}}$ & $0.79(0.74-0.83)^{\mathrm{a}}$ & $<0.001$ \\
\hline Fractional shortening $(\%)$ & $38 \pm 7$ & $40 \pm 6$ & $<0.001$ \\
\hline \multicolumn{4}{|l|}{ Left ventricular systolic function (qualitative) } \\
\hline Normal (\%) & 55.0 & 64.9 & $<0.001$ \\
\hline Fair $(\%)$ & 41.0 & 33.1 & \\
\hline Moderate or poor $(\%)$ & 3.9 & 2.1 & \\
\hline \multicolumn{4}{|l|}{ Diastolic parameters } \\
\hline Mitral valve inflow peak E $(\mathrm{m} / \mathrm{s})$ & $0.63 \pm 0.15$ & $0.66 \pm 0.16$ & $<0.001$ \\
\hline Mitral valve inflow peak A (m/s) & $0.72 \pm 0.16$ & $0.80 \pm 0.17$ & $<0.001$ \\
\hline Mitral valve inflow deceleration time (ms) & $219 \pm 47$ & $211 \pm 45$ & $<0.001$ \\
\hline E/A ratio & $0.86(0.71-1.00)^{\mathrm{a}}$ & $0.81(0.70-1.00)^{\mathrm{a}}$ & $<0.001$ \\
\hline
\end{tabular}

Categorical variables are expressed as percentage. Continuous variables are expressed as mean \pm standard deviation. $T$-tests, chi square tests and Mann-Whitney tests were used where appropriate

${ }^{a}$ Median and inter-quartile range because of skewed distribution 
blood pressure generally showed positive associations, with the exception of an inverse association of age with left ventricular end diastolic dimension in women. Diastolic blood pressure showed inverse associations with left atrium diameter in men and women. Furthermore, total cholesterol showed associations with selected parameters (Supplementary Table 1). The age- and ultrasonography system adjusted results (data not shown) demonstrated additional associations between HDL-cholesterol and diabetes mellitus and most structural parameters (inverse and positive associations, respectively), which disappeared after further adjustment.

In Table 3, multivariable-adjusted associations with fractional shortening are displayed. Age, BMI and diastolic blood pressure showed inverse associations with fractional shortening, while systolic blood pressure showed positive associations. In men, the association of systolic blood pressure with fractional shortening did not reach statistical significance. However, the magnitude of the regression coefficient was comparable to that of the significant regression coefficient in women. The results were similar when models were adjusted for age- and ultrasonography system only (data not shown). Table 4 shows associations of

Table 3 Multivariable adjusted regression coefficients for risk factors, describing the increase in fractional shortening $\left(\% * 10^{-2}\right)$ per (sex-specific) standard deviation increase of the risk factors

\begin{tabular}{lc}
\hline Variable & Fractional shortening $\left(\% * 10^{-2}\right)$ \\
\hline Men & $-125(-163,-87.1)^{*}$ \\
Age (years) & $-39.4(-74.4,-4.34)^{*}$ \\
Body mass index $\left(\mathrm{kg} / \mathrm{m}^{2}\right)$ & $34.3(-4.94,73.6)$ \\
Systolic blood pressure $(\mathrm{mmHg})$ & $-54.3(-93.9,-14.6)^{\dagger}$ \\
Diastolic blood pressure $(\mathrm{mmHg})$ & $6.11(-27.7,39.9)$ \\
Total cholesterol (mmol/l) & $20.0(-14.0,53.9)$ \\
HDL-cholesterol $(\mathrm{mmol} / \mathrm{l})$ & $-51.8(-148.6,45.0)$ \\
Diabetes mellitus & $-41.8(-124,40.6)$ \\
Current smoking & $-77.5(-106,-49.6)^{*}$ \\
Women & $-26.1(-52.0,-0.13)^{*}$ \\
Age (years) & $36.4(5.52,67.3)^{*}$ \\
Body mass index $\left(\mathrm{kg} / \mathrm{m}^{2}\right)$ & $-47.6(-77.3,-17.8)^{\dagger}$ \\
Systolic blood pressure $(\mathrm{mmHg})$ & $10.3(-14.3,34.9)$ \\
Diastolic blood pressure $(\mathrm{mmHg})$ & $3.59(-21.2,28.3)$ \\
Total cholesterol (mmol/l) & $9.65(-63.1,82.4)$ \\
HDL-cholesterol (mmol/l) & $-32.9(-99.5,33.7)$ \\
Diabetes mellitus & \\
Current smoking &
\end{tabular}

All models are adjusted (where appropriate) for ultrasonography system, age, BMI, systolic and diastolic blood pressure, total and HDL cholesterol, diabetes mellitus, smoking, use of diuretics, betablockers, ACE inhibitors, lipid lowering drugs and heart rate

* $0.01<P<0.05,{ }^{\dagger} 0.001<P<0.01,{ }^{\ddagger} P<0.001$
Table 4 Population characteristics according to left ventricular systolic function, multivariable adjusted

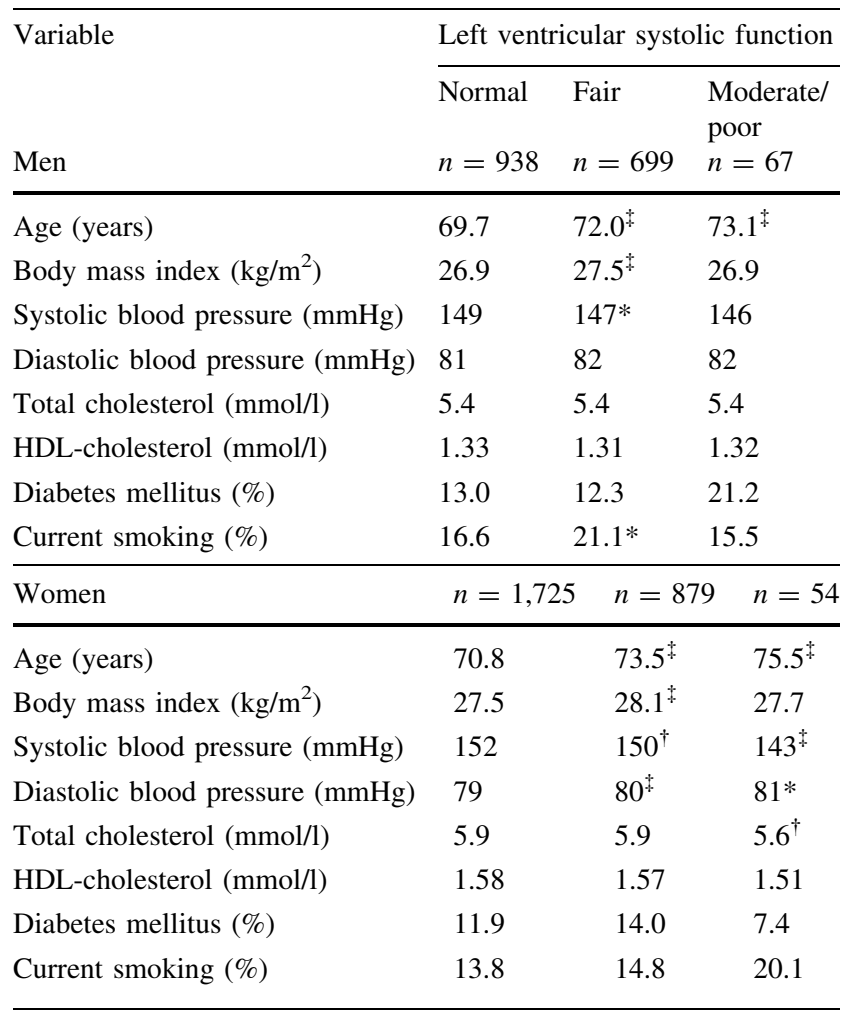

All models are adjusted (where appropriate) for ultrasonography system, age, BMI, systolic and diastolic blood pressure, total and HDL cholesterol, diabetes mellitus, smoking, use of diuretics, betablockers, ACE inhibitors, lipid lowering drugs and heart rat

$* 0.01<P<0.05,{ }^{\dagger} 0.001<P<0.01,{ }^{*} P<0.001$, compared to normal left ventricular systolic function

risk factors with left ventricular systolic function. Overall, the results were in accordance with Table 3. Age was higher in men and women with fair and moderate/poor function than in those with normal function. BMI was also significantly higher in men and women with fair function. Systolic blood pressure was lower in men with fair function than in men with normal function. In women, systolic blood pressure decreased and diastolic blood pressure increased according to systolic function categories. In Table 5, structural echocardiographic parameters are displayed according to categories of left ventricular systolic function. In general, left ventricular end-systolic and end-diastolic dimensions increased with worsening systolic function, as did interventricular septum thickness, left ventricular posterior wall thickness and left ventricular mass. As expected, fractional shortening significantly decreased with worse qualitative systolic function.

Subsequently associations between cardiovascular risk factors and diastolic echocardiographic parameters were examined. Age and diastolic blood pressure were inversely and significantly associated with E/A ratio in men and 
Table 5 Structural echocardiographic parameters and fractional shortening according to left ventricular systolic function, multivariable adjusted

\begin{tabular}{|c|c|c|c|}
\hline \multirow{2}{*}{$\begin{array}{l}\text { Variable } \\
\text { Men }\end{array}$} & \multicolumn{3}{|c|}{ Left ventricular systolic function } \\
\hline & $\begin{array}{l}\text { Normal } \\
n=938\end{array}$ & $\begin{array}{l}\text { Fair } \\
n=699\end{array}$ & $\begin{array}{l}\text { Moderate/ poor } \\
n=67\end{array}$ \\
\hline Diameter of left atrium (mm) & 42 & 42 & 43 \\
\hline Left ventricular end-systolic dimension $(\mathrm{mm})^{\S}$ & 30 & $35^{*}$ & $43^{*}$ \\
\hline Left ventricular end-diastolic dimension (mm) & 52 & $54^{*}$ & $59^{\ddagger}$ \\
\hline Interventricular septum thickness $(\mathrm{mm})^{\S}$ & 8.7 & $8.4^{\dagger}$ & 8.3 \\
\hline Left ventricular posterior wall thickness $(\mathrm{mm})^{\S}$ & 7.9 & $7.5^{*}$ & 7.9 \\
\hline Left ventricular mass $(\mathrm{g})^{\S}$ & 157 & 159 & $190^{*}$ \\
\hline Fractional shortening $(\%)$ & 42 & $35^{*}$ & $25^{+}$ \\
\hline Women & $n=1,725$ & $n=879$ & $n=54$ \\
\hline Diameter of left atrium (mm) & 39 & 39 & $40^{*}$ \\
\hline Left ventricular end-systolic dimension $(\mathrm{mm})^{\S}$ & 28 & $32^{*}$ & $40^{*}$ \\
\hline Left ventricular end-diastolic dimension (mm) & 49 & $50^{*}$ & $55^{*}$ \\
\hline Interventricular septum thickness $(\mathrm{mm})^{\S}$ & 8.1 & 8.0 & 8.1 \\
\hline Left ventricular posterior wall thickness $(\mathrm{mm})^{\S}$ & 7.3 & $7.2^{\dagger}$ & $7.8^{\dagger}$ \\
\hline Left ventricular mass $(\mathrm{g})^{\S}$ & 129 & 130 & $165^{*}$ \\
\hline Fractional shortening (\%) & 42 & $36^{*}$ & $26^{\ddagger}$ \\
\hline
\end{tabular}

All models are adjusted for ultrasonography system, age, BMI, systolic and diastolic blood pressure, total and HDL cholesterol, diabetes mellitus, smoking, use of diuretics, beta-blockers, ACE inhibitors, lipid lowering drugs and heart rate

$* 0.01<P<0.05,{ }^{\dagger} 0.001<P<0.01,{ }^{\dagger} P<0.001$, compared to normal left ventricular systolic function

$\S$ Geometric mean because of skewed distribution

women, whereas systolic blood pressure showed no association (Supplementary Table 2). BMI was positively and significantly associated with E/A ratio in women; in men, the regression coefficient was of similar magnitude but did not reach significance. Age- and ultrasonography system adjusted analyses also showed inverse associations between BMI and systolic blood pressure and E/A ratio in both sexes; however, these did not persist after additional adjustment. Categorization of E/A ratio confirmed the associations of lower E/A ratio with higher age and diastolic blood pressure in both sexes (Table 6). Of note is that it also showed significantly lower systolic blood pressure in women with $\mathrm{E} / \mathrm{A}$ ratio $<0.75$.

All above-mentioned analyses were adjusted for ultrasonography system to account for any possible differences. When running analyses in duplicate using the two systems separately, the associations were similar in direction and magnitude, but were more likely to reach statistical significance using the Acuson system.

\section{Discussion}

Summarizing, in this study, we have examined structural, systolic and diastolic echocardiographic parameters and their associations with cardiovascular risk factors in a population-based cohort. Structural parameters were generally larger in men, and larger values were most consistently associated with higher values of age, BMI and systolic blood pressure in both sexes. Although we had excluded participants with prevalent heart failure, myocardial infarction, and atrial fibrillation and flutter, moderate or poor left ventricular systolic function was found to be present in the participants, albeit with a modest prevalence (a total of $3.9 \%$ of men and $2.1 \%$ of women). Higher age, higher BMI, lower systolic and higher diastolic blood pressure were most consistently associated with worse systolic function. Furthermore, persons with worse systolic function had larger ventricular dimensions and larger left ventricular mass. With regard to diastolic parameters, we found that Doppler peak E and peak A velocities were larger in women than in men, but that $\mathrm{E} / \mathrm{A}$ ratio was lower in women than in men. Higher age and higher diastolic blood pressure were most consistently associated with lower E/A ratio in both sexes.

Strengths of the present study include its populationbased nature, its large size, and its standardized assessment of risk factors and echocardiographic characteristics. Nevertheless, several issues warrant consideration. First, we used global left ventricular function assessed qualitatively from two-dimensional images. This approach is easily obtainable and reflects the routine procedure in echocardiography 
Table 6 Population characteristics according to E/A ratio, multivariable adjusted

\begin{tabular}{|c|c|c|c|}
\hline \multirow{2}{*}{$\begin{array}{l}\text { Variable } \\
\text { Men }\end{array}$} & \multicolumn{3}{|l|}{ E/A ratio } \\
\hline & $\begin{array}{l}\mathrm{E} / \mathrm{A}<0.75 \\
n=457(27.4 \%)\end{array}$ & $\begin{array}{l}0.75 \leq \mathrm{E} / \mathrm{A} \leq 1.50 \\
n=1,183(70.9 \%)\end{array}$ & $\begin{array}{l}\mathrm{E} / \mathrm{A}>1.50 \\
n=29(1.7 \%)\end{array}$ \\
\hline Age (years) & $73.5^{+}$ & 69.7 & 69.2 \\
\hline Body mass index $\left(\mathrm{kg} / \mathrm{m}^{2}\right)$ & 27.2 & 27.2 & 27.2 \\
\hline Systolic blood pressure $(\mathrm{mmHg})$ & 147 & 148 & 149 \\
\hline Diastolic blood pressure $(\mathrm{mmHg})$ & $82^{\dagger}$ & 81 & 80 \\
\hline Total cholesterol (mmol/l) & 5.4 & 5.4 & 5.2 \\
\hline HDL-cholesterol (mmol/l) & 1.31 & 1.32 & 1.33 \\
\hline Diabetes mellitus (\%) & 14.7 & 12.1 & 16.0 \\
\hline Current smoking (\%) & 29.9 & 18.0 & 11.3 \\
\hline Women & $n=824(31.8 \%)$ & $n=1,730(70.9 \%)$ & $n=36(1.4 \%)$ \\
\hline Age (years) & $74.6^{*}$ & 70.4 & 69.7 \\
\hline Body mass index $\left(\mathrm{kg} / \mathrm{m}^{2}\right)$ & 27.9 & 27.6 & 26.9 \\
\hline Systolic blood pressure $(\mathrm{mmHg})$ & $149^{\dagger}$ & 151 & 152 \\
\hline Diastolic blood pressure $(\mathrm{mmHg})$ & $81^{*}$ & 79 & 77 \\
\hline Total cholesterol (mmol/l) & 6.0 & 5.9 & 5.7 \\
\hline HDL-cholesterol (mmol/l) & $1.55^{*}$ & 1.59 & 1.59 \\
\hline Diabetes mellitus $(\%)$ & 11.6 & 12.6 & 21.9 \\
\hline Current smoking (\%) & 14.6 & 14.4 & 13.0 \\
\hline
\end{tabular}

All models are adjusted (where appropriate) for ultrasonography system, age, BMI, systolic and diastolic blood pressure, total and HDL cholesterol, diabetes mellitus, smoking, use of diuretics, beta-blockers, ACE inhibitors, lipid lowering drugs and heart rate

* $0.01<P<0.05,{ }^{\dagger} 0.001<P<0.01,{ }^{*} P<0.001$, compared to $0.75 \leq \mathrm{E} / \mathrm{A} \leq 1.50$

laboratories. Prior studies have reported that the reproducibility and accuracy of visual assessments are as good as those of quantitative methods [25, 26], and similar visual assessments have also been used in other studies such as the Framingham Study [13]. Associations of risk factors with qualitative left ventricular function and with quantitatively measured fractional shortening showed similar patterns, and this suggest that assessment of left ventricular systolic function was performed appropriately in our study.

Second, we used E/A ratio as an indicator of diastolic function, while measures such as pulmonary venous flow or Doppler tissue imaging are needed to further categorize diastolic function [27, 28]. We were not able to assess such additional measures because of constraints in examination time available per participant, inherent to large-scale population-based research. Several different recommendations have been issued for categorization of diastolic function based on E/A ratio [29, 30]. E/A ratio is usually categorized according to absolute cut-points based on its physiology. We based our categorization of E/A ratio on absolute cutpoints used in recent reports [3, 24].

Furthermore, we should bear in mind that some of the echocardiographic measurements displayed considerable variability. Inter-reader and intra-reader measurement variabilities for E/A ratio and deceleration time reflect the practical difficulties involved in estimating Doppler peak E velocity and Doppler peak A velocity. In contrast, intrareader and inter-reader variability of structural and systolic parameters was in a much lower range. Finally, when analyzing the Esaote and Acuson ultrasonography systems separately, similar results were found, but associations were more likely to reach statistical significance in the group where the Acuson system was used. Higher numbers of participants in the Acuson group may partly explain this. However, precision may have also played a part, since the Acuson system provided images of somewhat better resolution. In the latter case, the associations we found may in reality be even stronger.

Previously, fractional shortening has been measured in a subset of 2,267 participants of the Rotterdam Study [31]. In accordance with the present study, fractional shortening was found to be somewhat higher in women $(40 \pm 7 \%)$ than in men $(38 \pm 8 \%)$. However, no significant association was found with age.

In the present study, women on average had better left ventricular systolic function than men, and E/A ratio was found to be lower in women. This is in accordance with known gender differences in heart failure; systolic dysfunction is more common in men and diastolic dysfunction tends to occur more often in women. This is explained by 


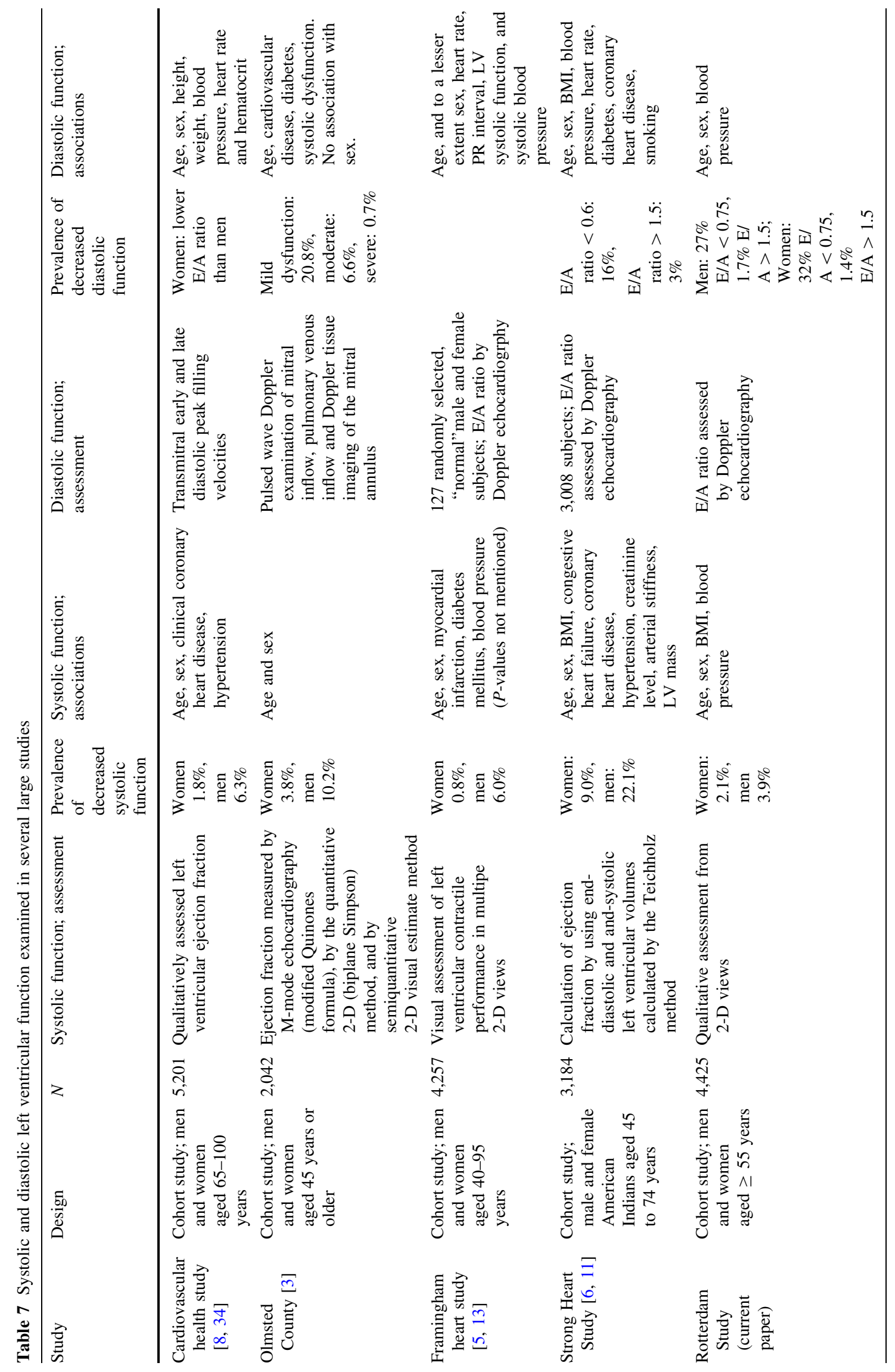


the fact that men have coronary artery disease as an underlying factor for heart failure more frequently than women, while women are more likely to have hypertension as an underlying factor [32, 33]. Heart failure that occurs after myocardial infarction is characterized by profound ventricular dilation, wall thinning, increased heart size, eccentric hypertrophy and systolic dysfunction. Heart failure that occurs due to long-standing hypertension is characterized by (concentric) left ventricular hypertrophy, increased myocardial mass and diastolic dysfunction, especially in the early stages.

The distribution of echocardiographic parameters and their associations with cardiovascular risk factors have previously been examined in several population-based studies. Table 7 provides an overview of the main findings of several large studies and compares them to the current study. It shows that prevalence of systolic and diastolic dysfunction varies across studies, probably in part due to differences in population characteristics and definitions of ventricular dysfunction. Nevertheless, associations of age, sex, and blood pressure with ventricular dysfunction appear to be rather consistent across studies.

In conclusion, we have found that moderate and poor left ventricular systolic function was present in asymptomatic men and women participating in the Rotterdam Study. With regard to diastolic parameters, we found that $\mathrm{E} / \mathrm{A}$ ratio was on average lower in women than in men. Higher age, BMI and diastolic blood pressure and lower systolic blood pressure were most consistently associated with worse systolic function in both sexes. Higher age and diastolic blood pressure were most consistently associated with lower E/A ratio in both sexes. Further research is warranted to investigate the value of echocardiography in identification of individuals with preclinical ventricular dysfunction.

Acknowledgments This study was supported by grant 2002B190 from the Netherlands Heart Foundation. The authors would like to thank Inge Haumersen, Saskia Hoornweg, Annemarieke Knetsch and Toos Stehmann for performing echocardiograms.

Open Access This article is distributed under the terms of the Creative Commons Attribution Noncommercial License which permits any noncommercial use, distribution, and reproduction in any medium, provided the original author(s) and source are credited.

\section{References}

1. Hunt SA, Abraham WT, Chin MH, Feldman AM, Francis GS, Ganiats TG, et al. ACC/AHA 2005 guideline update for the diagnosis and management of chronic heart failure in the adult: a report of the American College of Cardiology/American Heart Association Task Force on Practice Guidelines (Writing Committee to Update the 2001 Guidelines for the Evaluation and
Management of Heart Failure): developed in collaboration with the American College of Chest Physicians and the International Society for Heart and Lung Transplantation: endorsed by the Heart Rhythm Society. Circulation. 2005;112:e154-235.

2. Zile MR, Gaasch WH, Carroll JD, Feldman MD, Aurigemma GP, Schaer GL, et al. Heart failure with a normal ejection fraction: is measurement of diastolic function necessary to make the diagnosis of diastolic heart failure? Circulation. 2001;104: 779-82.

3. Redfield MM, Jacobsen SJ, Burnett JC Jr, Mahoney DW, Bailey KR, Rodeheffer RJ. Burden of systolic and diastolic ventricular dysfunction in the community: appreciating the scope of the heart failure epidemic. Jama. 2003;289:194-202.

4. Effect of enalapril on mortality and the development of heart failure in asymptomatic patients with reduced left ventricular ejection fractions. The SOLVD Investigators. N Engl J Med. 1992; 327:685-91.

5. Benjamin EJ, Levy D, Anderson KM, Wolf PA, Plehn JF, Evans JC, et al. Determinants of Doppler indexes of left ventricular diastolic function in normal subjects (the Framingham Heart Study). Am J Cardiol. 1992;70:508-15.

6. Devereux RB, Roman MJ, Paranicas M, Lee ET, Welty TK, Fabsitz RR, et al. A population-based assessment of left ventricular systolic dysfunction in middle-aged and older adults: the strong heart study. Am Heart J. 2001;141:439-46.

7. Devereux RB, Roman MJ, Palmieri V, Liu JE, Lee ET, Best LG, et al. Prognostic implications of ejection fraction from linear echocardiographic dimensions: the strong heart study. Am Heart J. 2003;146:527-34.

8. Gardin JM, Siscovick D, Anton-Culver H, Lynch JC, Smith VE, Klopfenstein HS, et al. Sex, age, and disease affect echocardiographic left ventricular mass and systolic function in the freeliving elderly. The cardiovascular health study. Circulation. 1995; 91:1739-48.

9. Xie X, Gidding SS, Gardin JM, Bild DE, Wong ND, Liu K. Left ventricular diastolic function in young adults: the coronary artery risk development in young adults study. J Am Soc Echocardiogr. 1995;8:771-9.

10. Aurigemma GP, Gottdiener JS, Shemanski L, Gardin J, Kitzman D. Predictive value of systolic and diastolic function for incident congestive heart failure in the elderly: the cardiovascular health study. J Am Coll Cardiol. 2001;37:1042-8.

11. Bella JN, Palmieri V, Roman MJ, Liu JE, Welty TK, Lee ET, et al. Mitral ratio of peak early to late diastolic filling velocity as a predictor of mortality in middle-aged and elderly adults: the strong heart study. Circulation. 2002;105:1928-33.

12. Lauer MS, Evans JC, Levy D. Prognostic implications of subclinical left ventricular dilatation and systolic dysfunction in men free of overt cardiovascular disease (the Framingham Heart Study). Am J Cardiol. 1992;70:1180-4.

13. Wang TJ, Evans JC, Benjamin EJ, Levy D, LeRoy EC, Vasan RS. Natural history of asymptomatic left ventricular systolic dysfunction in the community. Circulation. 2003;108:977-82.

14. Hofman A, Breteler MM, van Duijn CM, et al. The Rotterdam Study: 2010 objectives and design update. Eur J Epidemiol. 2009; 24:553-72.

15. Report of the expert committee on the diagnosis and classification of diabetes mellitus. Diabetes Care. 2003;26 Suppl 1:S5-20.

16. Bleumink GS, Knetsch AM, Sturkenboom MC, Straus SM, Hofman A, Deckers JW, et al. Quantifying the heart failure epidemic: prevalence, incidence rate, lifetime risk and prognosis of heart failure the Rotterdam Study. Eur Heart J. 2004;25: 1614-9.

17. van Bemmel JH, Kors JA, van Herpen G. Methodology of the modular ECG analysis system MEANS. Methods Inf Med. 1990; 29:346-53. 
18. Willems JL, Abreu-Lima C, Arnaud P, van Bemmel JH, Brohet $\mathrm{C}$, Degani R, et al. The diagnostic performance of computer programs for the interpretation of electrocardiograms. N Engl J Med. 1991;325:1767-73.

19. Kors JA, van Herpen G, Wu J, Zhang Z, Prineas RJ, van Bemmel $\mathrm{JH}$. Validation of a new computer program for Minnesota coding. J Electrocardiol. 1996;29(Suppl):83-8.

20. Henry WL, DeMaria A, Gramiak R, King DL, Kisslo JA, Popp $\mathrm{RL}$, et al. Report of the American society of echocardiography committee on nomenclature and standards in two-dimensional echocardiography. Circulation. 1980;62:212-7.

21. Schiller NB, Shah PM, Crawford M, DeMaria A, Devereux R, Feigenbaum $\mathrm{H}$, et al. Recommendations for quantitation of the left ventricle by two-dimensional echocardiography. American society of echocardiography committee on standards, subcommittee on quantitation of two-dimensional echocardiograms. J Am Soc Echocardiogr. 1989;2:358-67.

22. Devereux RB, Alonso DR, Lutas EM, Gottlieb GJ, Campo E, Sachs I, et al. Echocardiographic assessment of left ventricular hypertrophy: comparison to necropsy findings. Am J Cardiol. 1986;57:450-8.

23. Quinones MA, Otto CM, Stoddard M, Waggoner A, Zoghbi WA. Recommendations for quantification of Doppler echocardiography: a report from the Doppler quantification task force of the nomenclature and standards committee of the American society of echocardiography. J Am Soc Echocardiogr. 2002;15:167-84.

24. Ommen SR, Nishimura RA. A clinical approach to the assessment of left ventricular diastolic function by Doppler echocardiography: update 2003. Heart. 2003;89 Suppl 3:iii18-23.

25. Amico AF, Lichtenberg GS, Reisner SA, Stone CK, Schwartz RG, Meltzer RS. Superiority of visual versus computerized echocardiographic estimation of radionuclide left ventricular ejection fraction. Am Heart J. 1989;118:1259-65.

26. Jensen-Urstad K, Bouvier F, Hojer J, Ruiz H, Hulting J, Samad B, et al. Comparison of different echocardiographic methods with radionuclide imaging for measuring left ventricular ejection fraction during acute myocardial infarction treated by thrombolytic therapy. Am J Cardiol. 1998;81:538-44.

27. Garcia MJ, Thomas JD, Klein AL. New Doppler echocardiographic applications for the study of diastolic function. J Am Coll Cardiol. 1998;32:865-75.

28. Wang J, Nagueh SF. Current perspectives on cardiac function in patients with diastolic heart failure. Circulation. 2009;119: 1146-57.

29. How to diagnose diastolic heart failure. European study group on diastolic heart failure. Eur Heart J. 1998;19:990-1003.

30. Oh JK, Appleton CP, Hatle LK, Nishimura RA, Seward JB, Tajik AJ. The noninvasive assessment of left ventricular diastolic function with two-dimensional and Doppler echocardiography. J Am Soc Echocardiogr. 1997;10:246-70.

31. Mosterd A, Hoes AW, de Bruyne MC, Deckers JW, Linker DT, Hofman A, et al. Prevalence of heart failure and left ventricular dysfunction in the general population; the Rotterdam Study. Eur Heart J. 1999;20:447-55.

32. Hussey LC, Hardin S. Sex-related differences in heart failure. Heart Lung. 2003;32:215-23; quiz 224-5.

33. Petrie MC, Dawson NF, Murdoch DR, Davie AP, McMurray JJ. Failure of women's hearts. Circulation. 1999;99:2334-41.

34. Gardin JM, Arnold AM, Bild DE, Smith VE, Lima JA, Klopfenstein HS, et al. Left ventricular diastolic filling in the elderly: the cardiovascular health study. Am J Cardiol. 1998;82:345-51. 